\title{
Modelling the radiobiological effect of intraoperative X-ray brachytherapy for breast cancer using an air-filled spherical applicator
}

\author{
Kris Armoogum, MSc', Simon Evans, MSc', David Morgan, MB² \\ 'Department of Radiotherapy Physics, Royal Derby Hospital, Derby Teaching Hospitals NHS Foundation Trust, Uttoxeter Road, Derby, \\ ${ }^{2}$ Breast Services, Sherwood Forest Hospitals NHS Foundation Trust, Sutton-in-Ashfield, Nottingham, United Kingdom
}

\begin{abstract}
Purpose: We present a framework, in which we compare a conventional standard dose of 50 Gy in 25 fractions with accelerated partial breast irradiation (APBI) using electronic brachytherapy (eBT). We discuss how radiobiological modelling enables us to establish a framework, within which we can compare external beam radiotherapy (EBRT). This leads to a determination of the shell of isoeffect in breast tissue, at which very low $\mathrm{kV}$ eBT can be considered to be clinically equivalent to standard EBRT.

Material and methods: To estimate relative biological effectiveness (RBE) values as a function of dose and irradiation time, we used a modified linear quadratic (LQ) approach, taking into account the ability of this new device, to deliver 20 Gy at the surface of a $40 \mathrm{~mm}$ diameter rigid, hollow spherical applicator in less than 2 minutes. In this study, we considered the radiobiological effectiveness of the Papillon+TM X-ray brachytherapy device operating at $30 \mathrm{kV}$, $0.3 \mathrm{~mA}$ producing dose rates in excess of $14 \mathrm{~Gy} / \mathrm{min}$.

Results: Calculated clinical RBEs ranged from 1.154 at the surface of a $40 \mathrm{~mm}$ diameter applicator to 1.100 at $35 \mathrm{~mm}$ from the applicator surface for the Papillon+ device. The absolute physical dose D (abs) $30 \mathrm{kV}$ ranged from $20.00 \mathrm{~Gy}$ at the applicator surface to 1.20 at $35 \mathrm{~mm}$ distant. The product of the isoeffective single dose of ${ }^{60} \mathrm{Co}$ reference radiation - $(\mathrm{RBE})^{60} \mathrm{Co}$, and the RBE corrected standard 2 Gy equivalent dose fractions $\left(\mathrm{EQD}_{2}\right)$ doses, $\mathrm{EQD}_{2}(30 \mathrm{kV}){ }^{*}(\mathrm{RBE})^{60} \mathrm{Co}$ ranged from $98.62 \mathrm{~Gy}$ at the applicator surface to 1.13 at $35 \mathrm{~mm}$. The 'shell of isoeffect', the value on the X-axis where the $\mathrm{EQD}_{2}(30 \mathrm{kV}){ }^{*}(\mathrm{RBE})^{60} \mathrm{Co}$ line crosses the $50 \mathrm{~Gy}$ mark on the Y-axis, was found to be approximately $3.5 \mathrm{~mm}$ beyond the applicator surface.

Conclusions: The 'shell of isoeffect' can serve as a useful metric with which to compare the radiobiological effectiveness of low $\mathrm{kV}$ eBT with various regimes of conventional EBRT.
\end{abstract}

Key words: brachytherapy, breast cancer, radiobiology, X-ray.

\section{Purpose}

Accelerated partial breast irradiation in various forms is now often routinely used, and authoritative guidelines for its use have been published [1]. The acceleration of treatment time is at its most extreme in techniques of intraoperative radiotherapy (IORT), where a single fraction of radiation is delivered to the tumor bed immediately after resection of the cancer, before surgical closure. A large randomized trial, with permissive entry criteria, comparing one particular type of IORT (TARGIT-A) to external beam radiotherapy (EBRT) has reported comparable early results for local tumor control [2]. In a similar procedure using the Papillon+TM X-ray brachytherapy device, a hollow spherical applicator is placed temporarily in the surgical resection cavity; a kilovoltage $X$-ray tube, the tip of which lies at the center of the applicator, generates the radiation. This type of treatment will be called here 'electronic brachytherapy' (eBT).

To understand radiobiologically why eBT and EBRT should give comparable clinical results, requires complex three-dimensional analysis, as the distribution of the radiation dose in tissue around the radiotherapy applicator is markedly different for eBT compared with EBRT. In our calculations, we are considering a tumor, for which the American Society for Radiation Oncology (ASTRO) consensus guidelines would regard partial breast radiotherapy as an acceptable option, and many clinicians using EBRT would treat such a patient with a homogeneous whole breast dose of 50 Gy in 25 fractions with no tumor bed boost. This dose regime is taken as the stan-
Address for correspondence: Kris Armoogum, MSc, Department of Radiotherapy Physics, Royal Derby Hospital, Derby Teaching Hospitals NHS Foundation Trust, Uttoxeter Road, Derby DE22 3NE, United Kingdom, phone: +44 1332 787189, fax: +44 1332 786020, 凶 e-mail: kris.armoogum@nhs.net
Received: 03.03.2016

Accepted: 20.07.2016

Published: 24.08 .2016 
dard treatment [3]. The radiation dose in tissue following eBT is inhomogeneous, with a very high dose adjacent to the applicator surface, falling off rapidly with increasing distance. At some point, at a distance from the radiation source, there will be a transition from the higher biological effect of eBT compared to EBRT at short distances to the lower effect seen further away. At this point, the two treatments will be equally effective, so this can be referred to as a point of isoeffect, as previously described by Herskind et al. [4]. Having defined this point at a (one-dimensional) distance from the central point of radiation emission, its implications for overall tumor control probability (TCP) are here analyzed in a way that takes into account the three-dimensional reality of the tissues. We have based our calculations on a different $\alpha / \beta$ ratio for breast tumor control of 3.5 Gy [3].

With a hypothetical point source of radiation inside, a rigid, hollow spherical applicator embedded in homogeneous tissue, the point of isoeffect will occur at exactly the same distance from the radiation source in every direction, thus creating a three-dimensional contour that takes a spherical shape, and which we shall refer to as the shell of isoeffect. Within this shell, the eBT treatment will be more effective than EBRT, whereas outside it, the reverse is true. To compare the two treatments in terms of TCP, further factors must also be considered - the distribution and density of the biological targets of radiotherapy and their

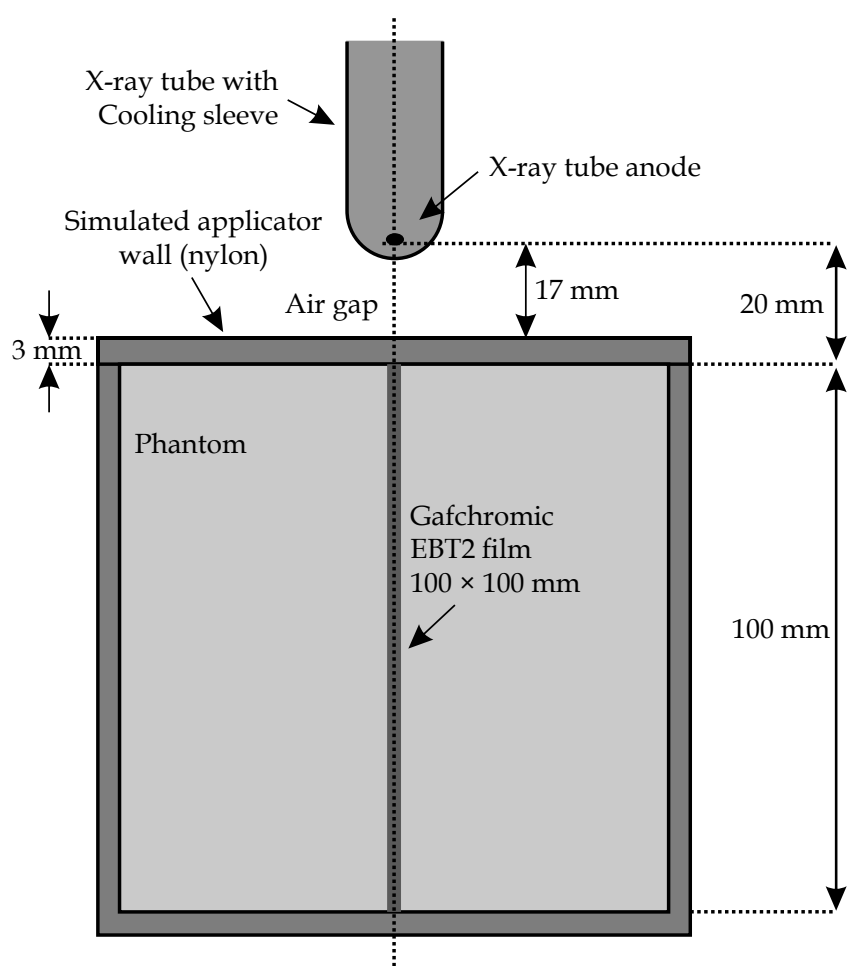

Fig. 1. Set-up used for depth dose measurements. Experimental set-up used to obtain depth-dose curves showing the X-ray source, custom-built water equivalent phantom (with a simulated nylon applicator wall) of dimensions $100 \mathrm{~mm} \times 100 \mathrm{~mm} \times 100 \mathrm{~mm}$. Gafchromic ${ }^{\circledR}$ EBT-2 dosimetry film (International Specialty Products, Wayne, NJ) was used to measure the dose at distances of 20 to $70 \mathrm{~mm}$ away from the tip of the probe, in increments of $1 \mathrm{~mm}$ variations within these volumes. If all the targets lie within the shell of isoeffect, the higher effectiveness of eBT here would make it, the more effective treatment (higher TCP). Whereas if the targets were scattered evenly throughout the whole breast, the small volume within the shell where eBT is more powerful, would be overwhelmed in comparison to the volume of breast tissue outside, and so EBRT would have the greater TCP. We propose that there could be a region, possibly a spherical volume, where the therapeutic effect of eBT and EBRT are equivalent.

\section{Material and methods}

\section{Measurement of depth dose data}

Depth doses were measured in a tissue equivalent phantom using Gafchromic ${ }^{\circledR}$ EBT-2 dosimetry film (International Specialty Products, Wayne, NJ, USA). The film was cut to $100 \mathrm{~mm} \times 100 \mathrm{~mm}$ and mounted within the phantom at right angles, along the central axis of the X-ray tube. Films were scanned 24 hours post-exposure using an Epson Expression 10000XL flatbed scanner. Scanning was performed in transmission mode with $150 \mathrm{dpi}$ with red channel data extracted and saved in uncompressed tagged image format (TIFF). As shown in Figure 1, the top of the Papillon+TM X-ray tube was mounted in a custom-built jig to leave a $20 \mathrm{~mm}$ gap between the anode and the proximal edge of the EBT-2 film. This gap equates to the space inside a hollow $40 \mathrm{~mm}$ diameter applicator, and consists of a $17 \mathrm{~mm}$ air gap and a $3 \mathrm{~mm}$ nylon layer to simulate the applicator wall. With an inherent filtration equivalent to $0.4 \mathrm{~mm} \mathrm{Al}$, the percentage depth dose falls to approximately $50 \%$ after traversing the $3 \mathrm{~mm}$ nylon layer. The phantom does not exactly reproduce the scatter geometry of the intended clinical setup and somewhat underestimates the absorbed dose at depth. Exposures were made for 5 seconds, and the films were scanned and analyzed using public domain image analysis software (ImageJ, National Institutes of Health, Bethesda, MD, USA).

\section{Relative biological effectiveness at low dose}

For any given geometry, the dose deposited by very low energy X-rays decreases with depth and the relative biological effectiveness (RBE) increases with decreasing dose. However, the increase in RBE is mitigated due to a $1-2 \%$ increase in beam hardening up to $10 \mathrm{~mm}$ deep in tissue [5]. Tissue attenuates low-energy X-rays more strongly than high energy ones, primarily because of photoelectric absorption. A heterogeneous beam traversing tissue becomes proportionately richer in high-energy photons, and hence more penetrating, or 'harder', with a lower RBE. Previous studies have found values of $\alpha_{H} / \alpha_{L}$ (low dose RBEs) in the range 0.97 to 1.17 for various cell lines irradiated with $X$-rays below $50 \mathrm{kV}$ $[6,7,8,9,10,11,12,13,14,15]$.

\section{Relative biological effectiveness calculations for high dose rate X-ray brachytherapy}

To estimate RBE values as a function of dose and irradiation time, we modified the Linear Quadratic (LQ) 
approach used by Brenner et al. [16]. In this study, we consider the radiobiological effectiveness of the Papillon+ ${ }^{\mathrm{TM}}$ X-ray brachytherapy (eBT) device (Ariane Medical Systems Ltd, Derby, UK) operated at $30 \mathrm{kV}, 0.3 \mathrm{~mA}$ with no added filtration delivering a single $20 \mathrm{~Gy}$ fraction to the surface of a $40 \mathrm{~mm}$ applicator in $<2$ minutes, compared with over 20 minutes needed by the Intrabeam ${ }^{\mathrm{TM}}$ for an applicator of the same diameter $[17,18]$.

From Brenner et al.,

$$
\begin{array}{r}
\operatorname{RBE}\left(D_{H}\right)=\frac{\gamma L}{2 G D_{H}}\left[\sqrt{1+\frac{4 G}{\gamma L}\left(\frac{\alpha_{H}}{\alpha_{L}} D_{H}+\frac{G}{\gamma L} D_{H}^{2}\right)^{2}}-1\right] \\
\text { Equation 1, }
\end{array}
$$

where $G$ is the generalized Lea-Catcheside time factor accounting for dose protraction during continuous irradiation at a constant dose rate for a delivery time $(T)$. The quantity $\gamma_{L}$ is the conventional low-LET $\alpha / \beta$ ratio for the tumor relapse in the breast, $\alpha_{H} / \alpha_{\mathrm{L}}$ equates to $\mathrm{RBE}_{\max }$ and $\mathrm{D}_{\mathrm{H}}$ is the prescribed dose. The quantity $\mathrm{T}_{1 / 2}$ is the halftime for sublethal cellular damage repair. In this study, we consider only mono-exponential repair kinetics based on the assumption of a single, dose-independent rate of repair of sublethal injury in the target cells. A range of values for the repair half times of acute or late responding tissues is demarcated with a reasonable fit to the clinical data. For the acute effects (or tumor control), the best fits are obtained for repair half times of about 30 minutes [14].

We have used $\mathrm{T}_{1 / 2}=30$ minutes as the halftime for cellular recovery from sublethal damage and with the Papillon ${ }^{\mathrm{TM}}$ able to deliver $20 \mathrm{~Gy}$ in a time $\mathrm{T}<2$ minutes, then we can state that $T_{«} T_{1 / 2}$ and $G$ tends to 1 . For tumor relapse in the breast $\alpha_{H} / \alpha_{L}$ equates to $R B E_{\text {max }}$, the maximum RBE as the dose approaches zero. Estimated low dose RBEs were adopted from Brenner et al. [16].

Equation 1 can be rewritten as:

$$
\operatorname{RBE}\left(D_{H}\right)=\frac{\gamma L}{2 D_{H}}\left[\sqrt{1+\frac{4 D_{H}}{\gamma L}\left(R B E_{\text {max }}+\frac{D_{H}}{\gamma L}\right)}-1\right]
$$

Equation 2

Showing that the RBE at any dose is a function of $\mathrm{RBE}_{\max } \alpha / \beta$ ratio and dose.

$\mathrm{D}_{\mathrm{H}}$ is the prescribed dose (PD) of $20 \mathrm{~Gy}$ to the surface of a $40 \mathrm{~mm}$ diameter rigid, hollow spherical applicator. A meta-analysis of START-A and the START pilot trial showed an adjusted estimate of $\alpha / \beta$ ratio for breast tumor control of 3.5 Gy (95\% CI: 1.2-5.7) and this is used in subsequent calculations (Figure 1).

\section{Point of isoeffect for breast cancer}

By correcting for the higher RBE of the eBT (Equation 1), and using the LQ model to adjust for fractionation differences, we have attempted to translate the depth dose curve of eBT into an equivalent dose (biologically equivalent dose) of conventional megavoltage radiotherapy given in $2 \mathrm{~Gy}$ fractions. For this calculation, an $\alpha / \beta$ ratio for breast cancer of $3.5 \mathrm{~Gy}$ is used, as suggested by the results of the START trial [3]. The point on the X-axis, at which the corrected curve crossed the $50 \mathrm{~Gy}$ point on the Y-axis, thus becomes a 'Point of Isoeffect' where we should expect the two schedules to have an equivalent radiobiological effect on breast cancer. Nearer the applicator, the eBT treatment will be more effective, and less so beyond this point. If the biological "targets" at which the radiation is aimed were mainly to be found within the few millimeters between the applicator surface and the 'Point of isoeffect', then the higher RBE of electronic brachytherapy in this area might at least balance the lower RBE achieved in the tissue beyond this point, where the target population density is likely to be less.

\section{Results}

\section{Depth doses for the Papillon+}

The percentage depth dose obtained from the Papillon+TM operating at $30 \mathrm{kV} 0.3 \mathrm{~mA}$ (inherent filtration $0.4 \mathrm{~mm} \mathrm{Al}$ ) for a typical $40 \mathrm{~mm}$ diameter applicator is shown in Figure 2. As there is no tissue within the applicator, the X-axis begins at the surface of the applicator and represents increasing depths in a tissue equivalent phantom. There will be the usual uncertainties associated with measuring depth doses using radiochromic film. Such as intra- and inter-film variations, scan reproducibility, and the uncertainty in experimental set-up. However, these are fully described elsewhere in the literature and are expected to contribute an overall error of the order of $\pm 5 \%$ [19].

\section{Shell of isoeffect for breast cancer}

Physical doses were calculated from the $30 \mathrm{kV}$ percentage depth dose in Figure 2, assuming a prescribed dose of $20 \mathrm{~Gy}$ in one fraction to the surface of a $40 \mathrm{~mm}$ diameter spherical applicator, and these were plotted on a semi-logarithmic graph as $\mathrm{D}(\mathrm{abs}) 30 \mathrm{kV}$. This was then converted to the corresponding isoeffective acute dose of ${ }^{60} \mathrm{Co}$ reference radiation by multiplying with the modelled RBEs at different depths. ${ }^{60}$ Co gamma rays were used as the reference radiation since over the depths of clinical interest, up to $20 \mathrm{~mm}$, the change in the ${ }^{60} \mathrm{Co}$ gamma ray

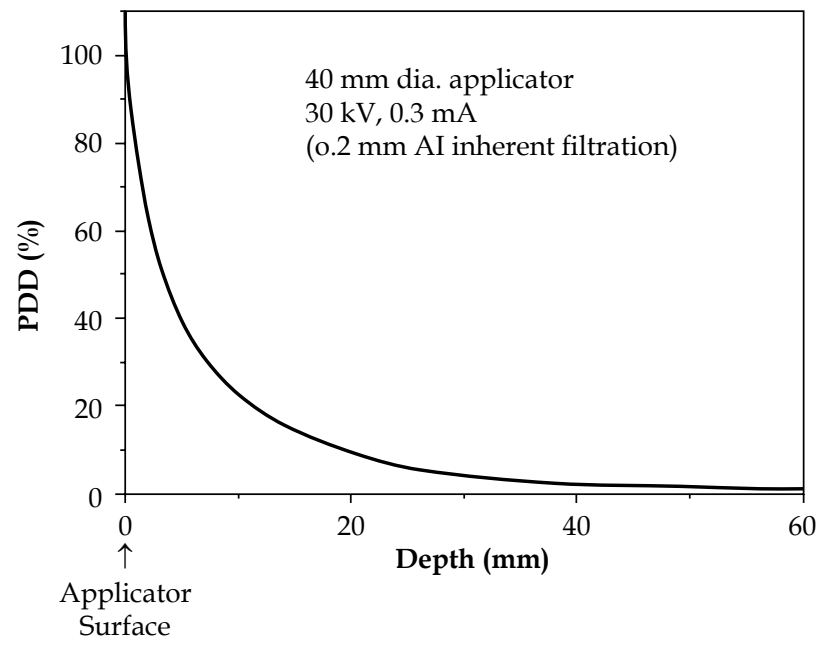

Fig. 2. Beam performance at $30 \mathrm{kV}$ with only inherent filtration closely mimics the Intrabeam ${ }^{\mathrm{TM}}$ device as used during the TARGIT-A trial, the rapid dose falls off being due to low mean beam energy in addition to inverse square law effects 
spectrum is negligible. Predicted low dose RBEs $\left(\alpha_{H} / \alpha_{L}\right)$ were adopted from Brenner et al. and used in Equation 1 to calculate the (RBE) ${ }^{60} \mathrm{Co}$ for the Papillon+TM device operating at $30 \mathrm{kV}$. These calculated clinical RBEs ranged from 1.154 at the surface of a $40 \mathrm{~mm}$ diameter applicator to 1.100 at $35 \mathrm{~mm}$ from the applicator surface. These are somewhat lower than those found by Reniers et al. who calculated the RBE for breast tissue to be in the range 0.89 to 1.43 . However, direct comparisons are difficult to make with a high degree of certainty due to large experimental uncertainties and how well microdosimetric mathematical modelling reflects real biological systems [20] (Table 1).

The product of the physical doses and the corresponding (RBE) ${ }^{60}$ Co gives the isoeffective acute doses $\mathrm{D}$ (iso)

Table 1. Predicted low dose relative biological effectiveness (RBEs) $\left(\alpha_{H} / \alpha_{L}\right)$ were adopted from Brenner [16] and used in Equation 1 to calculate the relative $\mathrm{RBE}{ }^{60} \mathrm{Co}$ for the Papillon $+^{\mathrm{TM}}$ device operating at $30 \mathrm{kV}$. These calculated clinical RBEs ranged from 1.154 at the surface of a $40 \mathrm{~mm}$ diameter applicator to 1.100 at $35 \mathrm{~mm}$ from the applicator surface

\begin{tabular}{lcc} 
Depth $(\mathrm{mm})$ & \multicolumn{2}{c}{ Versus ${ }^{60} \mathrm{Co}[\alpha / \beta=3.5 \mathrm{~Gy})$} \\
\cline { 2 - 3 } & $\alpha_{\mathrm{H}} / \alpha_{\mathrm{L}}$ & $(\mathrm{RBE}){ }^{60} \mathrm{Co}$ \\
\hline 0 & 3.05 & 1.154 \\
\hline 5 & 2.67 & 1.127 \\
\hline 10 & 2.54 & 1.118 \\
\hline 15 & 2.48 & 1.113 \\
\hline 20 & 2.44 & 1.110 \\
\hline 25 & 2.40 & 1.107 \\
\hline 30 & 2.35 & 1.104 \\
\hline 35 & 2.30 & 1.100 \\
\hline
\end{tabular}

Table 2. The product of the isoeffective single dose of ${ }^{60} \mathrm{Co}$ reference radiation, relative biological effectiveness (RBE) ${ }^{60} \mathrm{Co}$ and the RBE corrected standard 2 Gy equivalent dose fractions $\left(E Q D_{2}\right)$ doses, $\mathrm{EQD}_{2}(30 \mathrm{kV})$ * $\mathrm{RBE}{ }^{60} \mathrm{Co}$ ranged from $98.62 \mathrm{~Gy}$ at the applicator surface to 1.13 at $35 \mathrm{~mm}$

Depth $(\mathrm{mm}) \mathrm{D}_{(\mathrm{abs})} 30 \mathrm{kV}(\mathrm{RBE}){ }^{60} \mathrm{Co} \mathrm{EQD}_{2}(30 \mathrm{kV}) *{ }^{\mathrm{RBE}}{ }^{60} \mathrm{Co}$

\begin{tabular}{llll}
\hline 0 & 20.00 & 1.154 & 98.62 \\
\hline 5 & 12.00 & 1.127 & 38.11 \\
\hline 10 & 7.50 & 1.118 & 16.76 \\
\hline 15 & 4.60 & 1.113 & 7.54 \\
\hline 20 & 3.40 & 1.110 & 4.74 \\
\hline 30 & 1.60 & 1.104 & 1.64 \\
\hline 35 & 1.20 & 1.100 & 1.13
\end{tabular}

${ }^{60} \mathrm{Co}$. The LQ model was then used to convert the single isoeffective acute $\mathrm{D}$ (iso) ${ }^{60} \mathrm{Co}$ doses to the biologically equivalent total dose of conventional EBRT administered in $2 \mathrm{~Gy}$ fractions $\left(\mathrm{EQD}_{2}\right)$ as given by Equation 3.

$E Q D_{2 G y}=\left[D_{x} G y(x+\alpha / \beta)\right] /(2+\alpha / \beta) \quad$ Equation 3,

where $D_{x}$ is the total dose at a particular depth and $x$ is the dose per fraction. The $\mathrm{EQD}_{2}$ was then corrected for the decreasing RBE at depth to give $\mathrm{EQD}_{2}(30 \mathrm{kV}){ }^{*}(\mathrm{RBE}){ }^{60} \mathrm{Co}$ (Table 2).

As described above, the point of isoeffect can be read off as the value on the $\mathrm{X}$-axis where the $\mathrm{EQD}_{2}(30 \mathrm{kV})$ * (RBE) ${ }^{60} \mathrm{Co}$ line crosses the 50 Gy mark on the Y-axis, a distance (isoeffective depth) of approximately $3.5 \mathrm{~mm}$ beyond the applicator surface (Figure 3).

\section{Discussion}

We have established a framework within which to compare the biological effect of eBT (prescribed as $20 \mathrm{~Gy}$ at applicator surface) with EBRT (prescribed as 50 Gy in 25 fractions). Using this, we have calculated that for a $40 \mathrm{~mm}$ diameter rigid, hollow, spherical applicator radiobiological equivalence will occur at an approximate distance of $3.5 \mathrm{~mm}$ from the surface of the applicator. If we consider individual radiation beamlets, as each beamlet progresses into breast tissue, it will encounter an unknown concentration of targets along its path. The radial probability of destruction of targets is constant for all beamlets at a given distance in breast tissue that is, at the surface of a sphere. Combining such spheres, we have a range of probabilities forming concentric, minutely thin, radial probability shells of quasi-spherical outline. Each combination of applicator size and EBRT

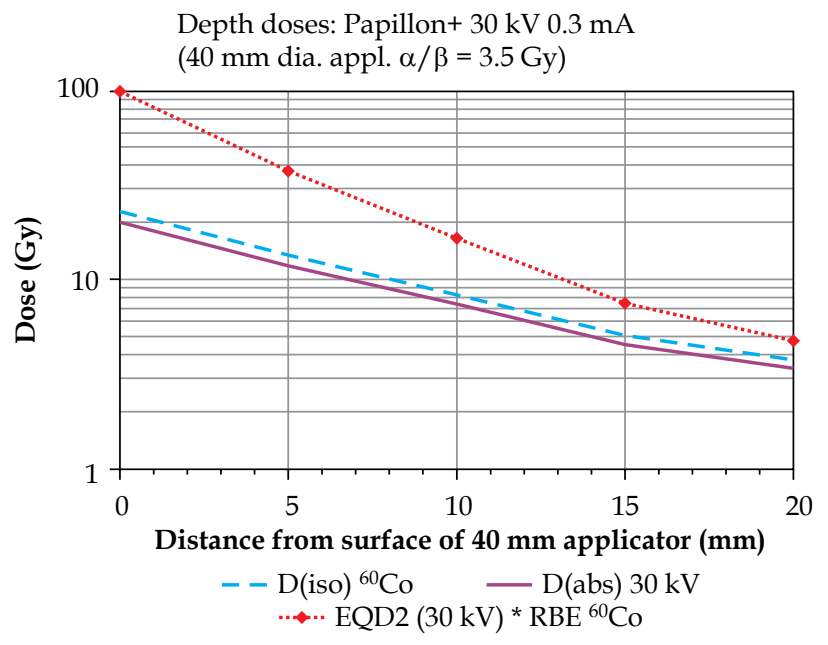

Fig. 3. Dose distribution for $30 \mathrm{kV} X$-rays generated by the Papillon ${ }^{\mathrm{TM}}$ with a $40 \mathrm{~mm}$ diameter spherical applicator. The absolute physical dose $\mathrm{D}(\mathrm{abs}) 30 \mathrm{kV}$, the isoeffective single dose of the ${ }^{60} \mathrm{Co}$ reference radiation, $\mathrm{D}($ iso $){ }^{60} \mathrm{Co}$, and the RBE corrected $\mathrm{EQD}_{2 \mathrm{~Gy}}$ doses, $\mathrm{EQD}_{2}(30 \mathrm{kV}){ }^{*} \mathrm{RBE}{ }^{60} \mathrm{Co}$ are shown as a function of distance from the surface of the applicator. $\mathrm{EQD}_{2}(30 \mathrm{kV}){ }^{*} \mathrm{RBE}{ }^{60} \mathrm{Co}$ accounts for the effect of RBE with distance from the applicator surface 
prescription regime could be expected to have a unique 'shell of isoeffect'. Against biological targets of radiotherapy that lie closer to the applicator surface than this shell, eBT will be more effective than EBRT, and less effective for those lying further out.

It now becomes important to consider what constitutes these "targets". In conventional radiobiology, these are considered to be viable tumor clonogens, and it is thought that these will have a physical distribution that coincides with the outlying tumor cells [21]. It was found that such cells regularly occurred at considerable distances from the edge of the main tumor mass, although in general, the shorter the distance, the more the cells. However, there are increasing reasons for thinking that there may be other targets of therapeutic radiation, which can broadly be considered as aspects of the tumor microenvironment.

It has been found that wound fluid stimulates the proliferation and invasion of breast cancer cell lines. However, this effect was almost completely absent when wound fluids from TARGIT-treated patients were used. Analysis showed that these fluids displayed an altered expression of some cytokines and failed to stimulate the activation of some intracellular signal transduction pathways.

It may be that IORT directly to the tumor bed alters the molecular composition and biological activity of surgical wound fluid from those patients. This could partially explain the very low recurrence rates found in a large pilot study using TARGIT [22].

The surgical act precipitates an avalanche of local biological responses, some of which may well enhance tumor growth, and these responses are likely to be at their maximum at the rim of the surgical cavity - the tissue that is in direct contact with the applicator used for eBT. This rim is indeed the locus of much of the "target" that is indirectly supported by recent guidelines published by ASTRO/SSO on the desirable width of tumor-free margin found on histological examination of the excision specimen [1]. On reviewing the available evidence, the authors of this guideline concluded that, as long as the cut edge (which equates to the "rim") was tumor-free, the depth of tumor-free margin beyond this did not influence outcomes after EBRT. This could be interpreted as indicating that the number of putative clonogens found lying in tissue within a few millimeters of the surgical rim has very little importance, and that the rim of the cavity itself is more important a target than the outlying cells. In turn, this implies that TCP is heavily dependent on the effectiveness of the radiation at very short distances from the applicator, as is the case with eBT. This could be an important factor in the non-inferiority of IORT to EBRT shown by the TARGIT-A Trial.

As breast cancers of different phenotypes express different biological behavior in various ways, this might influence how the biological targets were distributed in tissue. For tumors where the targets were mainly concentrated at the cavity rim, eBT would be expected to have a higher ultimate TCP than for those where they diffuse more extensively into adjacent tissue. Both TARGIT-A and the ELIOT trials give initial hints that some categories of breast cancer are more likely to be controlled by eBT than others [23]. The capability of the Papillon+TM to vary its X-ray energy (30 to $50 \mathrm{kVp}, 0$ to $3 \mathrm{~mA}$ ) and filtration might prove a fruitful area of future investigation in this respect. For example, at $30 \mathrm{kVp}$ and $0.20 \mathrm{~mm} \mathrm{Al}$ (half value layer), the Papillon ${ }^{\mathrm{TM}}$ mimics the Intrabeam and at $50 \mathrm{kVp}$ and $0.8 \mathrm{~mm} \mathrm{Al}$ added filtration it mimics treatment with ${ }^{192} \mathrm{Ir}$.

A further consideration in respect of biological targets that were humorally influenced, is that there would be a time, probably soon after surgery when they would be most potent, and this is when radiation therapy should occur. The better results seen in the TARGIT-A trial when IORT was used immediately after surgery rather than at a later date ("pre-" and "post-pathology" strata in the trial) supports this view.

\section{Conclusions}

We have presented a theoretical framework, which enables determination of the radiobiological equivalence of two different treatment modalities for breast cancer namely, X-ray brachytherapy and EBRT. In this study, we considered the radiobiological effectiveness of the Papillon+TM X-ray brachytherapy device operating at $30 \mathrm{kV}$, $0.3 \mathrm{~mA}$ using a $40 \mathrm{~mm}$ diameter rigid, hollow, spherical applicator, and producing dose rates in excess of $14 \mathrm{~Gy} /$ min at the applicator surface. For this case, radiobiological equivalence between eBT (prescribed as $20 \mathrm{~Gy}$ at applicator surface) and EBRT (prescribed as $50 \mathrm{~Gy}$ in 25 fractions) was found at approximately $3.5 \mathrm{~mm}$ distance from the surface of the applicator.

While the ultimate test of any new modality is its proof of effectiveness in well-designed clinical studies, the methodology used here in identifying a 'shell of isoeffect' may be useful in the clinical interpretation of trial results, and may possibly help gain further biological insights into the pathophysiological mechanisms involved. The methodology can also be readily transferred to calculations of predicted effects on important normal tissues such as skin, and to the effects that beam modification (changes in $\mathrm{kV}$ or filtration) and applicator size will have.

\section{Acknowledgements}

The Authors acknowledge the substantial contributions made by Prof. Bleddyn Jones and Prof. Roger Dale to this manuscript. DM and KSA met with Bleddyn and Roger at the Gray Institute for Radiation Oncology and Biology, University of Oxford in the autumn of 2012, and that meeting is where this paper was conceptualized.

\section{Disclosure}

Authors report no conflict of interest.

\section{References}

1. Moran M, Schnitt S, Giuliano A et al. Society of Surgical Oncology-American Society for Radiation Oncology consensus guideline on margins for breast-conserving surgery with whole-breast irradiation in stages I and II invasive breast cancer. Int J Radiat Oncol Biol Phys 2014; 88: 553-564. 
2. Vaidya J, Wenz F, Bulsara M et al. Risk-adapted targeted intraoperative radiotherapy versus whole-breast radiotherapy for breast cancer: 5-year results for local control and overall survival from the TARGIT-A randomised trial. Lancet 2014; 383: 603-613.

3. Haviland J, Agrawal R, Aird E et al. The UK START (Standardisation of Breast Radiotherapy) Trials: 10-year follow-up results. Cancer Res 2012; 72 (Suppl 24): S4-1.

4. Herskind C, Griebel J, Kraus-Tiefenbacher U et al. Sphere of Equivalence - A novel target volume concept for intraoperative radiotherapy using low-energy $\mathrm{x}$ rays. Int $\mathrm{J}$ Radiat Oncol Biol Phys 2008; 72: 1575-1581.

5. Ebert M, Carruthers B. Dosimetric characteristics of a low kV intra-operative $\mathrm{x}$-ray source: Implications for use in a clinical trial for treatment of low-risk breast cancer. Med Phys 2003; 30: 2424-2431.

6. Schmid E, Regulla D, Kramer H et al. The effect of $29 \mathrm{kVX}$ rays on the dose response of chromosome aberrations in human lymphocytes. Radiat Res 2002; 158: 771-777.

7. Lehnert A, Dörr W, Lessmann E et al. RBE of $10 \mathrm{kV}$ X rays determined for the human mammary epithelial cell line MCF-12A. Radiat Res 2008; 169: 330-336.

8. Hill M. The variation in biological effectiveness of X-rays and gamma rays with energy. Radiat Prot Dosimetry 2004; 112: 471-481.

9. Heyes G, Mill A, Charles M. Enhanced biological effectiveness of low energy $\mathrm{X}$-rays and implications for the UK breast screening programme. Br J Radiol 2006; 79: 195-200.

10. Spadinger I, Palcic B. The relative biological effectiveness of 60Co gamma-rays, $55 \mathrm{kV}$ X-rays, $250 \mathrm{kV}$ X-rays, and $11 \mathrm{MeV}$ electrons at low doses. Int J Radiat Biol 1992; 61: 345-353.

11. Raju M, Carpenter S, Chmielewski J et al. Radiobiology of ultrasoft X rays. I. Cultured hamster cells (V79). Radiat Res 1987; 110: 396-412.

12. Brenner D, Amols H. Enhanced risk from low-energy screenfilm mammography X rays. Br J Radiol 1989; 62: 910-914.

13. Depuydt J, Baert A, Vandersickel V et al. Relative biological effectiveness of mammography X-rays at the level of DNA and chromosomes in lymphocytes. Int J Radiat Biol 2013; 89: 532-538.

14. Beyreuther E, Dörr W, Lehnert A et al. Relative biological effectiveness of 25 and $10 \mathrm{kV} \mathrm{X-rays} \mathrm{for} \mathrm{the} \mathrm{induction} \mathrm{of} \mathrm{chro-}$ mosomal aberrations in two human mammary epithelial cell lines. Radiat Environ Biophys 2009; 48: 333-340.

15. Pop L, van den Broek J, Visser A et al. Constraints in the use of repair half times and mathematical modelling for the clinical application of HDR and PDR treatment schedules as an alternative for LDR brachytherapy. Radiother Oncol 1996; 38: 153-162.

16. Brenner D, Leu C, Beatty J et al. Clinical relative biological effectiveness of low-energy x-rays emitted by miniature x-ray devices. Phys Med Biol 1999; 44: 323-333.

17. Gérard J, Myint S, Croce O et al. Renaissance of contact x-ray therapy for treating rectal cancer. Expert Rev Med Devices 2011; 8: 483-492.

18. Armoogum K, Ackland C, Gardner J. Implementation and experiences of an intraoperative radiotherapy service. J Radiother Pract 2006; 5: 203-210.

19. Armoogum $K$, Watson C. A dosimetry intercomparison phantom for intraoperative radiotherapy. Z Med Phys 2007; 18: 120-127.

20. Reniers B, Liu D, Rusch T et al. Calculation of relative biological effectiveness of a low-energy electronic brachytherapy source. Phys Med Biol 2008; 53: 7125-7135.

21. Holland R, Veling S, Mravunac M et al. Histologic ultifocality of Tis, T1-2 breast carcinomas. implications for clinical trials of breast-conserving surgery. Cancer 1985; 56: 979-990.
22. Belletti B, Vaidya J, D'Andrea S et al. Targeted intraoperative radiotherapy impairs the stimulation of breast cancer cell proliferation and invasion caused by surgical wounding. Clin Cancer Res 2008; 14: 1325-1332.

23. Holmes D. Intraoperative radiotherapy in breast conserving surgery. J Surg Oncol 2014; 110: 68-74. 